\title{
AUTOMATIC NEAR-REAL TIME FLOOD EXTENT AND DURATION MAPPING BASED ON MULTI-SENSOR EARTH OBSERVATION DATA
}

\author{
Sandro Martinis, Marc Wieland, Michaela Rättich, Christian Böhnke, Torsten Riedlinger \\ German Aerospace Center, German Remote Sensing Data Center, Oberpfaffenhofen, 82234 Wessling, \\ Germany
}

\begin{abstract}
In order to support disaster management activities related to flood situations, an automatic system for near-real time mapping of flood extent and duration using multi-sensor satellite data is developed. The system is based on four processing chains for the automatic derivation of the inundation extent from Sentinel-1 and TerraSAR-X radar as well as from optical Sentinel-2 and Landsat data. While the systematic acquisition plan of the Sentinel-1/2 and Landsat satellites allows a continuous monitoring of inundated areas at an interval of a few days, the TerraSAR-X processing chain has to be triggered on-demand over the disasteraffected areas. Beyond flood extent masks, flood duration products are generated to indicate the temporal stability and evolution of flood events. The flood monitoring system is demonstrated on a severe flood situation in Mozambique related to cyclone Idai in 2019.
\end{abstract}

Index Terms - Flood Mapping, Flood Monitoring, Total Flood Duration, Backward Flood Duration, Flood Duration Quality, Sentinel-1/2, TerraSAR-X, Landsat

\section{INTRODUCTION}

Flooding is a widespread and frequently a dramatic natural disaster that affects lives, infrastructures, economics and ecosystems worldwide. Floods can cause loss of life and substantial property damage. Further, the economic ramifications of flood damage disproportionately impact the most vulnerable members of society. Emergency responders often request Earth Observation (EO) based crisis information for flood monitoring to target the often limited resources and to prioritize response actions during a disaster situation.

Single EO missions are in general not always suitable to fulfill the requirements of emergency responders with respect to revisit time and spatial coverage. Furthermore, disaster management especially benefits from the automation of algorithms to reduce time-consuming manual interactions of imaging experts in extracting crisis information from EO data. Therefore, an automatic multi-sensor satellite system for near-real time (NRT) flood extent mapping based on multi-sensor satellite data is presented. It consists of four automatic processing chains for the extraction of the inundation extent from Sentinel-1 (S-1) and TerraSAR-X radar as well as from optical Sentinel-2 (S-2) and Landsat EO data. In order to indicate the temporal stability of flooding over time, the flood system automatically computes two flood duration products, i.e. the Backward and Total Flood Duration, together with corresponding quality layers to indicate the temporal stability of an inundation over time. The multi-sensor EO-based flood monitoring system is demonstrated on a flood situation in Mozambique caused by the landfall of cyclone Idai in March 2019.

\section{EO-BASED MULTI-SENSOR FLOOD SYSTEM}

An overview of the multi-sensor flood monitoring system's workflow is visualized in Fig. 1. Due to the systematic data acquisition capability of S-1, the Sentinel-1 Flood Service $[5,2]$ has a key role within the proposed systematic NRT flood monitoring system. The two processing chains based on Landsat-8 and S-2 [6] complete the systematic monitoring capability of the system. A TerraSAR-X Flood Service [1] can be triggered on demand over a disaster area to increase the revisit period and/or to derive the inundation extent in higher detail than using the systematically acquiring sensors of up to a spatial resolution of $1 \mathrm{~m}$. All flood services contain the following generic steps: automatic EO data ingestion (S-1: Copernicus Open Access Hub and direct downlink at the ground station of DLR in Neustrelitz, S-2: Copernicus Open Acces Hub, TerraSAR-X: TerraSAR$\mathrm{X}$ delivery server, Landsat-8: USGS) and pre-processing, computation and adaption of global ancillary data (digital elevation models, topographic slope information and topographic indices, as well as reference water masks), automatic flood extent mapping, and dissemination of the crisis information, e.g. via a web-client. The radar-based processing chains make use of an automatic hierarchical tilebased thresholding approach in combination with fuzzylogic-based post-processing for the unsupervised extraction of the flood extent $[1,5]$. The thematic S-2 and Landsat services perform water segmentation with a convolutional neural network $(\mathrm{CNN})$ based on the U-Net architecture that 
has been trained, tested and validated on a global reference dataset representative for a variety of climatic, atmospheric and land-cover conditions. Clouds and cloud shadows are specifically handled by the network to remove potential biases from downstream analysis [6].

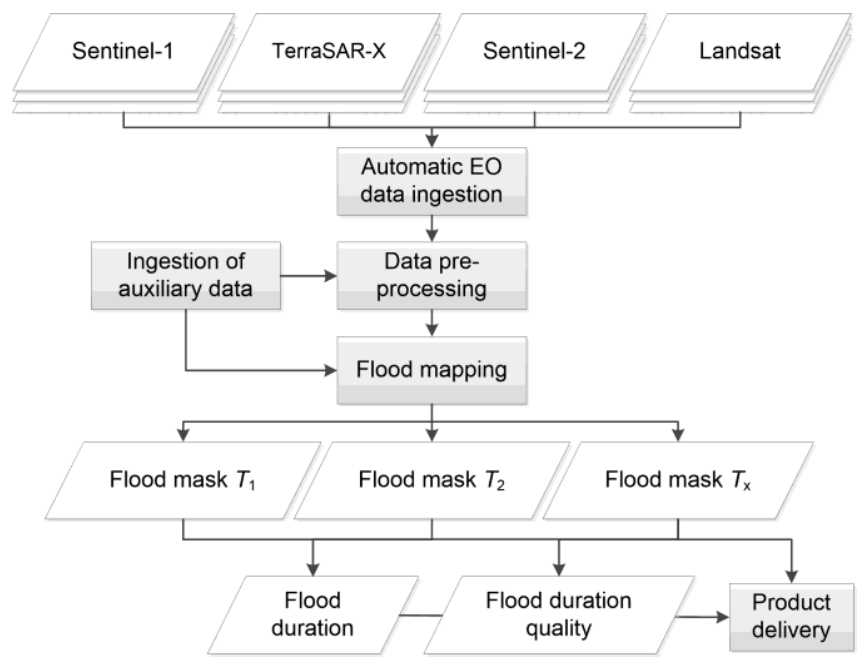

Figure 1: Workflow of the proposed EO-based system for flood extent mapping and flood duration estimation.

In order to provide emergency managers with spatiotemporal information about the stability and the evolution of an inundation event or to assess the damage related to flood events, the estimation of flood duration is a very crucial parameter. In order to estimate the flood duration on a regular basis in NRT in case of disaster situations a fully automated approach is developed [3] and integrated into the flood monitoring system.

Binary flood masks are automatically preprocessed including re-projection to WGS84, clipping to a defined AOI, merging (if there are several observations on the same day), and resampling to a common spatial resolution. The pre-processed flood masks serve as input for the subsequent processing where a pixel-based examination of the time series is carried out in order to compute two kinds of flood duration products: the Total Flood Duration (TFD) and the Backward Flood Duration (BFD). In addition, a Flood Duration Quality (FDQ) layer is generated in order to indicate the uncertainty related to both flood duration products. All available data sets are used for computing the flood duration products. In the case that a pixel is covered by cloud it is considered as no observation is available for this raster cell during the acquisition time.

BFD is computed for each image element backwards in time from the latest incoming satellite scene until the start of the inundation event. It is an indicator for the duration of an ongoing flood situation in days and should be delivered to the user in NRT in order to provide an indication of the persistency of the flooding. For each pixel the algorithm checks stepwise backwards in time until it is not covered by water any more. The BFD is updated as soon as more up-todate $\mathrm{EO}$ data gets available.

The TFD is computed for each pixel for a user-defined time period. In contrast to the BFD it might cover more than one flood event. The duration of $n$ flood events is summed in order to calculate the duration of flood coverage over time. The quality of the flood duration products depends on the observation frequency and temporal coverage of the used virtual satellite constellation.

The FDQ is automatically estimated to indicate the uncertainties related to these products. The computation takes the acquisition frequency and distribution of acquisitions during a flood event into account. By computing the BFD the uncertainty is expressed by the sum of the Pre-event (PreU) and Co-event Uncertainty (CoU). A third term, the Post-event Uncertainty (PostU), might be added by to PreU and $\mathrm{CoU}$ of each flood event in the defined time period by calculating the TFD. CoU is computed based on the number of gaps resulting from days without satellite observation between two days with flooding within a single inundation event. The influence of each gap on the uncertainty is weighted according to the duration of the gap. Thereby, long observation gaps get penalized with higher uncertainties.

The principle of the calculation of BFD, TFD, and FDQ is visualized for fictive flood events during a time period of 20 days $D_{\mathrm{x}}$ in Fig. 2.

(a)

\begin{tabular}{|l|l|l|l|l|l|l|l|l|l|l|l|l|l|l|l|l|l|l|l|}
\hline$D_{1}$ & $D_{2}$ & $D_{3}$ & $D_{4}$ & $D_{5}$ & $D_{6}$ & $D_{7}$ & $D_{8}$ & $D_{9}$ & $D_{10}$ & $D_{11}$ & $D_{12}$ & $D_{13}$ & $D_{14}$ & $D_{15}$ & $D_{10}$ & $D_{17}$ & $D_{18}$ & $D_{19}$ & $D_{20}$ \\
\hline
\end{tabular}
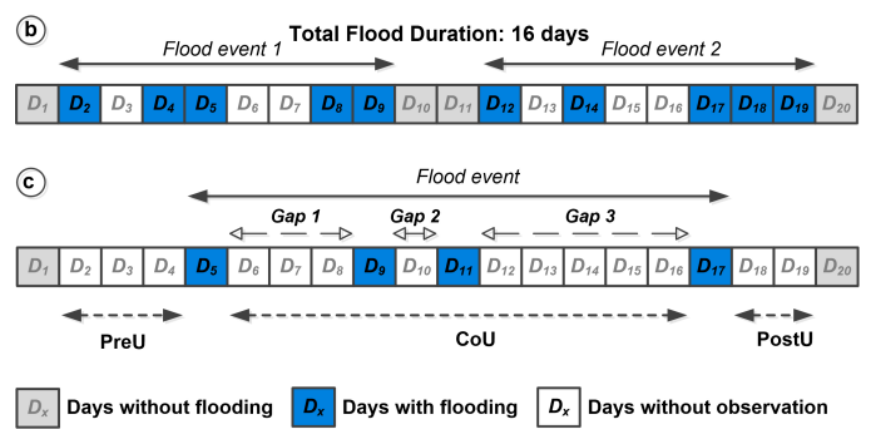

Figure 2: Schematic illustration for computing a) BFD, b) TFD, c) FDQ for a fictive flood scenario over a 20-day period.

\section{RESULTS}

\subsection{Study area and data set}

In this chapter, results of the EO-based flood system are presented for the flooding related to the tropical cyclone Idai in 2019, which formed over the Northern Mozambique Channel on March 9 and made landfall close to the city of Beira on the night of March 14 to 15 . The study area is 
mainly situated in the province Sofala and has an extent of $\sim 15,000 \mathrm{~km}^{2}$ (Fig. 3).

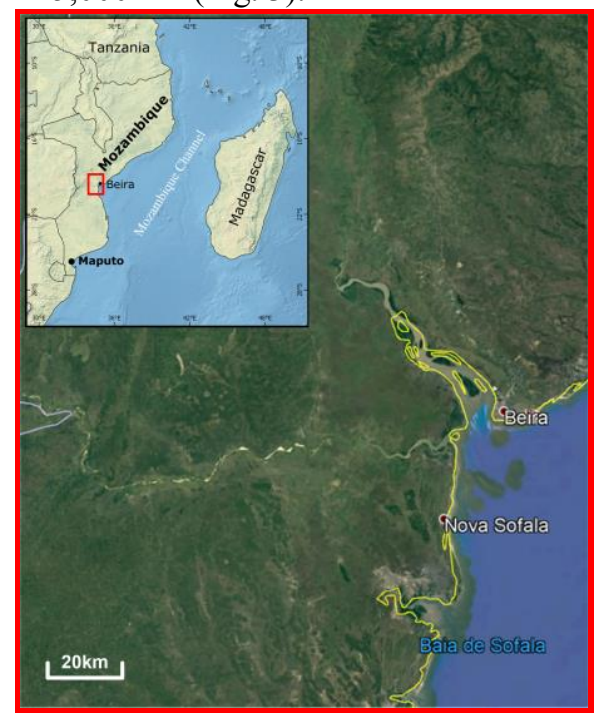

Figure 3: Study area in Mozambique. Map data (C) Google Earth and OpenStreetMap contributors.

In total $75 \mathrm{EO}$ data are used, which cover the area of interest (AOI) within the time 02/03/-07/04/2019:

- S-1: 33 Sentinel-1 GRD Interferometric Wideswath (IW) data acquired on 11 days (spatial resolution $\sim 20 \mathrm{~m}$ )

- S-2: 12 L1C Sentinel-2 scenes acquired on 12 days (36 tiles, spatial res. $10 \mathrm{~m}$ )

- TerraSAR-X: 4 ScanSAR data sets (spatial res. $18.5 \mathrm{~m}$ )

- Landsat-8: 2 scenes of the Operational Land Imager (OLI) onboard Landsat-8 (spatial res. 30m)

\subsection{Flood extent masks}

Due to the predicted landfall of Idai the International Charter "Space and Major Disasters" (https://disasterscharter.org/) was activated on March 14, 2019. Flood extent masks have been automatically derived from S-1, TerraSAR-X, S-2, and Landsat-8 data using the flood monitoring system. The 37-day period between $02 / 03 / 2019-07 / 04 / 2019$ is covered in total on 25 days by these EO missions. Due to the imaging capability irrespective of atmospheric conditions the radar missions S1 and TerraSAR-X allow a systematic monitoring of the flood extent and of the dynamic of the inundation. S-2 acquisitions are highly cloud covered on the days during/after the landfall. However S-2 data, together with Landsat-8, provide an additional value to monitor the recession of the water extent after the movement of the cyclone from 17/03/2019 onwards. Fig. 4 depicts examples of flood extent products based on S-1, TerraSAR-X, and S-2 data.

10 radar acquisitions (9 S-1 and 1 TerraSAR-X data) cover the whole AOI and are therefore used to calculate the dynamic of the flooding over the whole period. S-1-based results on 14/03/2019 (03:09 UTC) show that also before the landfall of Idai in the night of March 14 a significant part of the AOI is flooded ( $\left.682 \mathrm{~km}^{2}\right)$. On 19/03/2019 (Fig. 4a) the flood peak is reached where more than $1,800 \mathrm{~km}^{2}$ are inundated. These flooding occurred mainly in the catchment area of River Búzi in the west of the AOI. Between 20/03/$25 / 03 / 2019$ the flood extent drastically receded by more than $1,400 \mathrm{~km}^{2}$ to $400 \mathrm{~km}^{2}$. The recession of water occurred mainly in the central and southern parts of the AOI, whereas inundations persisted in the North. In the following time period until 07/04/2019 the flooding decreased slowly by $\sim 300 \mathrm{~km}^{2}$ to an extent of $\sim 105 \mathrm{~km}^{2}$. Due to the spatial resolution of the used EO data and the side-looking imaging geometry of SAR systems flooded areas might be underestimated in urban areas.

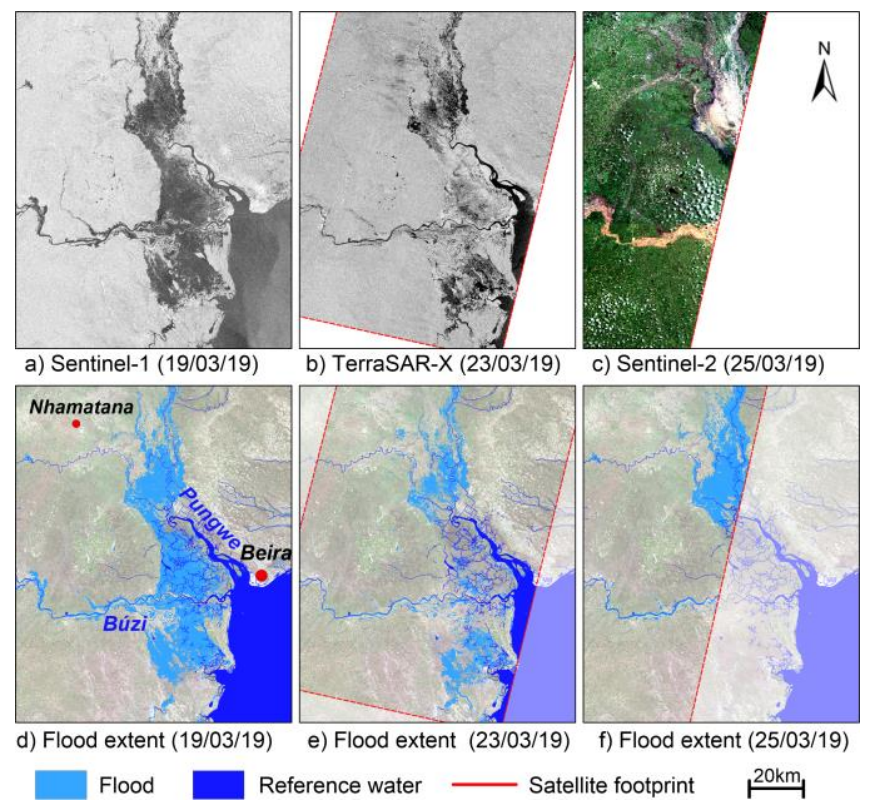

Figure 4: Results of the automatic multi-sensor flood system in Mozambique: a) S-1 of 19/03/2019 (C) ESA); b) TerraSAR-X of 23/03/2019 (C) DLR); c) S-2 (RGB: Red, Green, Blue) of 25/03/2019 (C) ESA) and d-f) derived single-temporal flood extents and reference water masks from Shuttle Radar Topography Mission Water Body Data (SWBD) [4] and data of HOT. A mosaic of S-2 tiles acquired on $02 / 12 / 2012$ serves as background in d-f. Modified from Rättich et al. (2020).

\subsection{Flood duration products}

The proposed flood monitoring system is able to automatically generate the TFD and BFD products as well as a corresponding FDQ in NRT based on flood masks derived from the respective flood services. In Fig. 5a the BFD is visualized computed at the peak of the flooding on $19 / 03 / 2019$. At this stage especially the central and southern part of the AOI is already flooded since $\sim 7$ days. This shows that regions have already been flooded some days before the landfall of Idai. This product can be used as indicator for the 
duration of an ongoing flood event and should be assessable for emergency responders in NRT in order to provide information about the persistency of an ongoing flood situation. The TFD is depicted for the observation period 02/03/2019 - 07/04/2019 in Fig. 5b. It considers all flooded pixels during this time period. Therefore, this product shows a larger inundation extent than the BFD. Fig. 5b shows extensive inundations of 14-20 days in the southern and northern part (along Pungwe river) of the AOI. In total an area of $\sim 2,500 \mathrm{~km}^{2}$ was inundated during 02/03/2019$07 / 04 / 2019$. More than $1,000 \mathrm{~km}^{2}$ were flooded on only $1-2$ days and $>1,700 \mathrm{~km}^{2}$ less than one week (1-7 days). Other regions were inundated longer - more than $600 \mathrm{~km}^{2}$ for $1-2$ weeks and $\sim 130 \mathrm{~km}^{2}$ for 2-3 weeks. The TFD can be used as damage indicator for insurance companies providing climate risk insurance products or be useful for long-term comparisons of annually recurring hydrological phenomena. The FDQ for the TFD product is shown in Fig. 5c. It shows relative low uncertainty values. Due to the high temporal coverage of EO acquisitions during this disaster, relative high certainty values between 1 and 19 are achieved for most regions. Higher uncertainty values are mainly related to the occurrence of clouds on one or more acquisition dates of the optical data, which cause a lower availability of valid pixels and the prolongation of observation gaps.

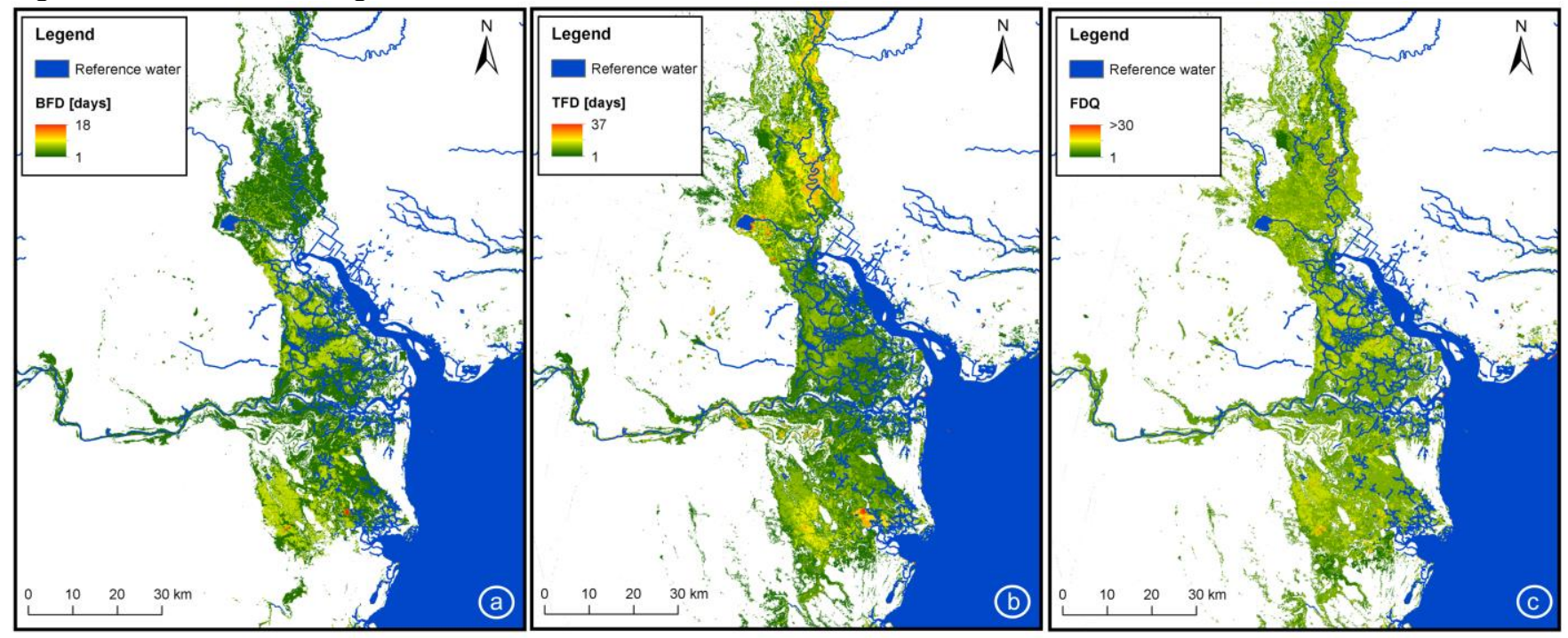

Figure 5: Flood duration products over the AOI in Mozambique derived from Sentinel-1, TerraSAR-X, Sentinel-2, and Landsat-8 data: a) BFD (02/03/2019-19/03/2019), TFD (02/03/2019-07/04/2019), and FDQ for the TFD (02/03/201907/04/2019). The reference water extent is based on SWBD [4] and data of HOT. Modified from Rättich et al. (2020).

\section{CONCLUSION}

In this study an automatic system for NRT flood extent and duration mapping based on multi-sensor EO imagery is presented. Flood masks are automatically derived using flood services based on S-1, S-2, TerraSAR-X, and Landsat8 data. Two different flood duration products are computed in order to provide 1) NRT information about the duration of an ongoing inundation and 2) the total duration of flood coverage for a pre-defined time period. Furthermore, a Flood Duration Quality layer is computed, which gives information about the reliability of the flood duration products by taking into account pre-event, co-event and, post-event uncertainties. The proposed system is demonstrated on a flood situation related to cyclone Idai in March 2019 in Mozambique.

\section{REFERENCES}

[1] Martinis, S., J. Kersten, and A. Twele, "A fully automated TerraSAR-X based flood service", ISPRS Journal of
Photogrammetry and Remote Sensing, vol. 104, pp. 203-212, 2015.

[2] Martinis, S., S. Plank, and K. Cwik, "The use of Sentinel-1 time-Series data to improve flood Monitoring in arid areas", Remote Sensing, vol. 10 (582), pp. 1-13, 2018.

[3] Rättich, M., S. Martinis, M. Wieland, "Automatic flood duration estimation based on multi-sensor satellite data. Remote Sensing, vol. 12, no. 4, pp. 1-19, 2020.

[4] SWBD, "Shuttle radar topography mission water body data set”, $\quad$ http://www.dds.cr.usgs.gov/srtm/version2_1/SWBD/ (accessed 09.01.2020).

[5] Twele, A., W. Cao, S. Plank, and S. Martinis, "Sentinel-1 based flood mapping: a fully automated processing chain," International Journal of Remote Sensing, 2016, vol. 37, no. 13, pp. 2990-3004, 2016.

[6] Wieland, M., S. Martinis, "A modular processing chain for automated flood monitoring from multi-spectral satellite data," Remote Sensing, vol. 11, no. 9, pp. 1-23, 2019. 